\title{
Sexual dimorphism in hepatic lipids is associated with the evolution of metabolic status in mice
}

\author{
Ana Francisca Soares ${ }^{1}$ [D । Jonathan Paz-Montoya ${ }^{2}$ | Hongxia Lei ${ }^{3}$ | Marc Moniatte ${ }^{2}$ | \\ Rolf Gruetter ${ }^{1,3}$
}

${ }^{1}$ École Polytechnique Fédérale de Lausanne, Laboratory for Functional and Metabolic Imaging (LIFMET), Lausanne, VD, Switzerland

${ }^{2}$ École Polytechnique Fédérale de Lausanne, Proteomics Core Facility (PCF), Lausanne, VD, Switzerland

${ }^{3}$ Center for Biomedical Imaging (CIBM), Lausanne VD, Switzerland and University of Geneva, Department of Radiology, Geneva, Switzerland

Correspondence

A. F. Soares, Ecole Polytechnique Federale de Lausanne, Laboratory for Functional and Metabolic Imaging (LIFMET), Lausanne VD, Switzerland.

Email: francisca.soares@epfl.ch

Funding information

Center for Biomedical Imaging (CIBM); Leenaards and Louis-Jeantet Foundations
Ectopic lipid accumulation in the liver is implicated in metabolic disease in an age- and sex-dependent manner. The role of hepatic lipids has been well established within the scope of metabolic insults in mice, but has been insufficiently characterized under standard housing conditions, where age-related metabolic alterations are known to occur. We studied a total of 10 male and 10 female mice longitudinally. At 3, 7 and 11 months of age, non-invasive ${ }^{1} \mathrm{H}$-magnetic resonance spectroscopy $\left({ }^{1} \mathrm{H}-\mathrm{MRS}\right)$ was used to monitor hepatic lipid content (HLC) and fatty acid composition in vivo, and glucose homeostasis was assessed with glucose and insulin challenges. At the end of the study, hepatic lipids were comprehensively characterized by nuclear magnetic resonance (NMR) and liquid chromatography-mass spectrometric analyses of liver tissue samples. In males, HLC increased from $1.4 \pm 0.1 \%$ at 3 months to $2.9 \pm 0.3 \%$ at 7 months $(p<0.01)$ and 2.7 $\pm 0.3 \%$ at 11 months $(p<0.05)$, in correlation with fasting insulin levels $(p<0.01, r=0.51)$ and parameters from the insulin tolerance test (ITT; $p<0.001, r=-0.69$ versus area under the curve; $p<0.01, r=-0.57$ versus blood glucose drop at $1 \mathrm{~h}$ post-ITT; $p<0.01, r=0.55$ versus blood glucose at $3 \mathrm{~h}$ post-ITT). The metabolic performance of females remained the same throughout the study, and HLC was higher than that of males at 3 months $(2.7 \pm 0.2 \%, p<0.01)$, but comparable at 7 months $(2.2 \pm 0.2 \%)$ and 11 months $(2.2 \pm 0.1 \%)$. Strong sexual dimorphism in bioactive lipid species, including diacylglycerols (higher in males, $p<0.0001$ ), phosphatidylinositols (higher in females, $p<0.001$ ) and omega-3 polyunsaturated fatty acids (higher in females, $p<0.01$ ), was found to be in good correlation with metabolic scores at 11 months. Therefore, in mice housed under standard conditions, sex-specific composition of bioactive lipids is associated with metabolic protection in females, whose metabolic performance was independent of hepatic cytosolic lipid content.

\section{KEYWORDS}

${ }^{1} \mathrm{H}-\mathrm{MRS}$, insulin resistance, LC-MS, lipidomics, liver fat, sexual dimorphism

\section{I INTRODUCTION}

Obesity is known to be associated with diseases in which severe metabolic dysregulation is present, including cardiovascular disease, cancer and type 2 diabetes. The metabolic risk is further aggravated by aging-associated adiposity ${ }^{1-3}$ in a gender-dependent manner. For instance, even though women generally display a proportionally higher amount of adipose tissue than men, they are at a lower metabolic risk. This feature may be

\footnotetext{
Abbreviations used: AAC, area above the curve; AGC, automatic gain control; ANOVA, analysis of variance; AUC, area under the curve; Cer, ceramide; DAG, diacylglycerol; ELISA, enzyme-linked immunosorbent assay; GlcCer, glucosylceramide; HLC, hepatic lipid content; HR-NMR, high-resolution, liquid-state, nuclear magnetic resonance; ITT, insulin tolerance test; LC-MS, liquid chromatography-mass spectrometry; LBPA, lyso-bis-phosphatidic acid; MCL, mean chain length; MRS, magnetic resonance spectroscopy; OGTT, oral glucose tolerance test; PG, glycerophosphoglycerol; PKC, protein kinase C; PtdCho, phosphatidylcholine; PtdEt, phosphatidylethanolamine; Ptdlns, phosphatidylinositol; PtdSer, phosphatidylserine; PUFA, polyunsaturated fatty acid; QUICKI, quantitative insulin sensitivity check index; RF, radiofrequency; SEM, standard error of the mean; SFA, saturated fatty acid; SLBPA, semi-lyso-bis-phosphatidic acid; SM, sphingomyelin; STEAM, stimulated echo acquisition mode; TG, triglyceride; UFA, unsaturated fatty acid; VAPOR, variable power RF pulses with optimized relaxation delays
} 
explained by sex-specific fat distribution that favors subcutaneous versus visceral depots in women, thereby offering them protection against metabolic disease. , $^{2,4}$

Dietary, surgical and genetic manipulations in male rodents have shown that inflammation, visceral fat and hepatic steatosis are strong determinants shifting obesity towards metabolic disease. ${ }^{5-7}$ In particular, hepatic steatosis may lead to insulin resistance independent of weight gain. ${ }^{8}$ Given the strong relationship between visceral fat and hepatic steatosis, ${ }^{9,10}$ sexual dimorphism in hepatic fat storage probably plays a role in the susceptibility to metabolic disease. Accordingly, the prevalence of non-alcoholic fatty liver disease is lower in women than men, ${ }^{11}$ and post-prandial hepatic lipid storage is greater in male than in female volunteers. ${ }^{12}$ Epidemiological and clinical experimental findings are reflected in rodent studies, demonstrating that females are protected against hepatic steatosis and related metabolic dysregulation in models of diet-induced obesity $^{13-15}$ and also in aging mice. ${ }^{16}$

Laboratory rodents with limited space and free access to food may be considered as natural models of a sedentary lifestyle ${ }^{17}$ and, indeed, show features of aging-associated obesity and metabolic disease. ${ }^{5}$ However, the contribution of hepatic lipids to metabolic dysregulation in mice housed under standard conditions has been poorly addressed and is likely to exhibit sexual dimorphism. Thus, in this study, we monitored possible changes in hepatic lipid content (HLC) and fatty acid composition (throughout the article, the designation 'fatty acid' refers to fatty-acyl chains in lipid molecules rather than to free fatty acids) over several months in mice housed under standard conditions. These longitudinal measurements were performed with ${ }^{1} \mathrm{H}$-magnetic resonance spectroscopy $\left({ }^{1} \mathrm{H}\right.$-MRS) in vivo, a powerful technique that has been used to monitor both lipid accumulation ${ }^{18-20}$ and depletion. ${ }^{21}$ In order to examine the specific relationship between hepatic lipids and the anticipated sex differences in metabolic performance, we performed glucose and insulin challenges throughout the study. Lipidomics data from high-resolution, liquid-state, nuclear magnetic resonance (HR-NMR) and liquid chromatography-mass spectrometry (LC-MS) analysis of liver samples collected at the end of the study provided complementary information on the complete hepatic lipid profile.

\section{I MATERIALS AND METHODS}

\section{1 | Animals}

Experiments were performed with the approval of the local ethics committee (Service de la consummation et des affaires vétérinaires, Epalinges, Switzerland). Male $(N=10)$ and female $(N=10)$ C57BL/6J mice (Charles River Laboratories, L'Arbresle, France) were housed, five per cage, in a 12-h light/12-h dark cycle (lights on at 07:00 h) with free access to water and a chow diet (Kliba-Nafag, Kaiseraugst, Switzerland). The diet was composed of $18.8 \%(\mathrm{~m} / \mathrm{m})$ protein, $5.6 \%(\mathrm{~m} / \mathrm{m})$ fat (from soybean oil) and $54.6 \%(\mathrm{~m} / \mathrm{m})$ nitrogen-free extract. After at least 1 week of acclimatization, mice were studied at 3, 7 and 11 months of age, with the ${ }^{1} \mathrm{H}$-MRS in vivo and metabolic tests performed as described below. At 11 months, mice were sacrificed by cervical dislocation under isoflurane anesthesia, and the livers were removed, freeze-clamped in liquid nitrogen-cooled metal tongs and stored at $-80^{\circ} \mathrm{C}$ until further processing.

\section{2 | Metabolic tests}

Oral glucose tolerance tests (OGTTs) were performed after at least a 3-day resting period following the MR session (described below). Six-hour fasted mice (food removed at $\sim 07: 30 \mathrm{~h}$ ) received a glucose load of $1.5 \mathrm{~g} / \mathrm{kg}$ by oral gavage, and blood glucose was monitored from the tail tip with a glucometer (Breeze, Bayer, Zürich, Switzerland) for $2 \mathrm{~h}$. The area under the curve (AUC) in the glucose versus time plot was calculated to assess glucose clearance from the blood. An insulin tolerance test (ITT) was then performed after a minimum 3-day resting period by intraperitoneal injection of insulin (0.75 IU/kg, Humulin, Novo Nordisk, Bagsvaerd, Denmark, diluted in $0.9 \% \mathrm{NaCl})$. Blood glucose was monitored from the tail tip for $3 \mathrm{~h}$ and the area above the curve (AAC) was calculated for the glucose versus time plots. Commercially available enzyme-linked immunosorbent assay (ELISA) kits were used to measure fasting insulin (Mercodia, Uppsala, Sweden) and C-peptide (Crystal Chem, Downers Grove, IL, USA) levels in the plasma. Insulin sensitivity was estimated with the quantitative insulin sensitivity check index $(\mathrm{QUICKI})$ as the inverse of the log 10 sum of fasting insulin $(\mu \mathrm{IU} / \mathrm{mL})$ and fasting glucose $(\mathrm{mg} / \mathrm{dL})$.

\section{3 | HLC and fatty acid composition by ${ }^{1} \mathrm{H}-\mathrm{MRS}$ in vivo}

Mice were anesthetized with isoflurane (4\% for induction, and 1-2\% afterwards, in 70 : 30 air : oxygen) and placed in an in-house-built holder. Body temperature was continuously monitored by a rectal probe and maintained at $36-37^{\circ} \mathrm{C}$ with a circulating warm water system. The respiratory rate was kept at $60-90 \mathrm{bpm}$ by the regulation of isoflurane delivery, and was continuously monitored with a pneumatic pillow through an MR-compatible system (SA Instruments, Stony Brook, NY, USA), which also delivered the necessary triggering signals for respiratory gating during the MR acquisitions. Mice were scanned in the supine position in a 26-cm, horizontal bore, 14.1-T magnet interfaced to a VNMRS DirectDrive console (Varian, Palo Alto, CA, USA). A ${ }^{1} \mathrm{H}$ quadrature surface coil (two 13-mm inner diameter physically decoupled loops) placed over the abdomen was used as transreceiver. Automated shimming to reduce magnetic field inhomogeneities was performed with FAST(EST)MAP. ${ }^{22}$ Multi-slice gradient echo images were acquired in the sagittal, axial and coronal orientations (flip angle, $85^{\circ}$; field of view, $25 \times 25$ mm ${ }^{2}$; data matrix, $128 \times 128$; and minimum TR and TE) for the identification of the liver and definition of a $2 \times 2 \times 2$-mm ${ }^{3}$ volume of interest for 
${ }^{1} \mathrm{H}-\mathrm{MRS}$. HLC was determined from ${ }^{1} \mathrm{H}-\mathrm{MR}$ spectra acquired with the stimulated echo acquisition mode (STEAM) (TE = 8 ms; mixing time, 20 ms; $\mathrm{TR}=6.5 \mathrm{~s} ; 4096$ complex points, 32 scans) as the peak area of the methylene protons at $1.3 \mathrm{ppm}$ (Lip 1.3) relative to that of the water plus Lip 1.3, with corrections for $T_{2}$ as described previously. ${ }^{21}$ The fatty acid composition of intra-hepatic lipids was characterized as described previously ${ }^{21}$ using indices for saturation, number of double bonds, unsaturated fatty acids (UFA), saturated fatty acids (SFA), polyunsaturated fatty acids/polyunsaturated bonds per chain (PUFA), polyunsaturated bonds per UFA, monounsaturated fatty acids (MUFA) and mean chain length $(\mathrm{MCL}$ ) (Table 1). These indices were obtained from different lipid resonances in spectra acquired with STEAM (TE = $2.8 \mathrm{~ms} ; \mathrm{mixing}$ time, $20 \mathrm{~ms}$; TR = $5 \mathrm{~s} ; 2048$ complex points; 86-128 scans), in combination with VAPOR [variable power radiofrequency (RF) pulses with optimized relaxation delays] water suppression and outer volume saturation. ${ }^{23}$ Individual spectra were corrected for $B_{0}$ drift and phase, and summed, with peak integration performed using LCModel ${ }^{24}$ version 6.3-1E with SPTYPE liver-11 and automatic metabolite simulation. The MR experiments, including animal setup, MR imaging, shimming and ${ }^{1} \mathrm{H}-\mathrm{MRS}$, lasted for about $1 \mathrm{~h}$.

\section{4 | Liver tissue extraction and lipid characterization by HR-NMR in vitro}

About 200 mg of lyophilized liver tissue were treated with chloroform-methanol $(2: 1, \mathrm{v} / \mathrm{v})$ for lipid extraction. ${ }^{25}$ The organic phase was dried under a nitrogen atmosphere to prevent oxidation. Dried lipids were dissolved in $500 \mu \mathrm{L}$ of chloroform-d for HR-NMR analysis. Fully relaxed ${ }^{1} \mathrm{H}-\mathrm{NMR}$ spectra of lipid extracts were acquired in a 600-MHz (14.1-T) DRX-600 spectrometer (Bruker BioSpin, Fällanden, Switzerland) equipped with a cryoprobe, and were obtained with 16 scans of a pulse-acquire sequence using a pulse delay of $10 \mathrm{~s}$ and an acquisition time of $2.5 \mathrm{~s}$. The equations in Table 1 were used to characterize total lipids extracted from the liver. As a result of the presence of highly unsaturated fatty acids, with three or more double bonds, ${ }^{21}$ the equation for MUFA is not applicable to liver extracts. In the HR-NMR spectra, the contribution of omega-3 PUFA was calculated from the resonance of the respective terminal methyl groups (Lip 0.9omega-3), which is shifted downfield relative to the non-omega-3 methyl groups (Lip 0.9), as shown below ${ }^{26}$ :

$$
\text { omega-3 PUFA }(\%)=\frac{\text { Lip 0.9omega-3 }}{\text { Lip } 0.9+\text { Lip 0.9omega-3 }} \times 100
$$

Lipid samples were recovered from the NMR tubes and the solvent was removed by evaporation under a nitrogen atmosphere.

\section{5 | Lipidomics by LC-MS}

Standards and solvents are listed in Supporting Information Table S1. Standards were dissolved in chloroform-methanol (1 : 1, v/v) at a concentration of $1 \mathrm{mM}$ and stored at $-20^{\circ} \mathrm{C}$. Dried lipid samples were re-dissolved in chloroform-methanol $(1: 1, v / v)$ and total phosphate content was determined to calculate the injection volume corresponding to $0.4 \mathrm{nmol}$ of phosphate. Separation was performed on a HILIC Kinetex column $\left(2.6 \mu \mathrm{m}, 2.1 \times 50 \mathrm{~mm}^{2}\right.$ ) on a Shimadzu Prominence UFPLC xr system (Tokyo, Japan). Mobile phase A was acetonitrile-methanol (10 : 1 , v/v) containing $10 \mathrm{mM}$ ammonium formate and $0.5 \%$ formic acid. Mobile phase B was deionized water containing $10 \mathrm{mM}$ ammonium formate and $0.5 \%$ formic acid. Gradient flow elution at $200 \mu \mathrm{L} / \mathrm{min}$ began at $5 \%$ B with a linear increase to $50 \%$ B over 4 min; $50 \%$ B was held for 1.5 min; lastly, the column was re-equilibrated for $2.5 \mathrm{~min}$.

Data were acquired in full scan mode at high resolution on a hybrid Orbitrap LTQ-XL (Thermo Fisher Scientific, Bremen, Germany). The system was operated at 60000 resolution $(\mathrm{m} / \mathrm{z} 400)$ with an automatic gain control (AGC) set at $5.0 \times 10^{5}$ and one microscan set at 250 ms maximum injection time. The heated electrospray source (HESI II) was operated in positive mode at a temperature of $100^{\circ} \mathrm{C}$ and a source voltage of 4.0 $\mathrm{kV}$. Sheath gas and auxiliary gas were set at 20 and 5 arbitrary units, respectively, whereas the transfer capillary temperature was set to $275^{\circ} \mathrm{C}$.

TABLE 1 Fatty acid composition indices derived from ${ }^{1} \mathrm{H}$-magnetic resonance $\left({ }^{1} \mathrm{H}\right.$-MR) spectra of hepatic lipids

\begin{tabular}{|c|c|}
\hline Saturation index & $\frac{\text { Lip } 1.3 \times 3}{\text { Lip } 0.9 \times 2}$ \\
\hline Unsaturated fatty acids (UFA) & $\frac{\text { Lip } 2.1 \times 3}{\text { Lip } 0.9 \times 4}$ \\
\hline Saturated fatty acids (SFA) & 1 - UFA \\
\hline Polyunsaturated fatty acids or polyunsaturated bonds per fatty acid (PUFA) & Lip $2.8 \times 3$ \\
\hline Mean chain length (MCL) & $\frac{1 / 2(\operatorname{Lip} 1.3+\operatorname{Lip} 2.1+\operatorname{Lip} 2.8)+\operatorname{Lip} 5.3 \& 5.2}{1 / 3 \times \operatorname{Lip} 0.9}$ \\
\hline
\end{tabular}

Chemically distinct fatty-acyl protons are identified by their chemical shift in the ${ }^{1} \mathrm{H}-\mathrm{MR}$ spectrum; for example, Lip 1.3 refers to the methylene protons at $1.3 \mathrm{ppm}$.

${ }^{\mathrm{a}} \mathrm{A}$ correction factor of 1.15 [1/(1 - 0.13)] was used to account for an underestimation of approximately $13 \%$ of the number of double bonds under our acquisition parameters. ${ }^{21}$ 
Mass spectrometric data were acquired with LTQ Tuneplus 2.5 and analyzed with Xcalibur 2.1 (Thermo Fisher Scientific). Lipid identification was carried out with Lipid Data Analyzer II (LDA v. 2.5.1, IGB-TUG Graz University, Graz, Austria). ${ }^{27}$ This algorithm identifies peaks by their respective retention time, $\mathrm{m} / \mathrm{z}$ and intensity. In order to minimize the number of potential elemental assignments, care was taken to calibrate the instrument regularly to ensure a mass accuracy consistently lower than 3 ppm.

The total amount of phosphate was similar in the lipid extracts from male and female livers (17.93 \pm 0.6 and $17.02 \pm 2 \mathrm{nmol} / \mathrm{mg}$, respectively), thereby allowing for a direct qualitative comparison of lipid species abundance between samples of both sexes. An initial coarse evaluation of the differences between sexes was performed by comparing the total signal area of each lipid class on the total ion chromatogram. The most abundant lipid classes were further evaluated by comparing the relative distribution of individual species identified by Lipid Data Analyzer II within the class. The contribution of individual species is expressed as the percentage ratio of their extracted ion chromatogram area versus the summed area of all lipid species identified in the class, in a similar manner to Rouzer et al. ${ }^{28}$ Only species contributing to more than $1 \%$ of the total signal of the class in at least one of the sexes were considered.

\section{6 | Statistics}

The reported values are the mean \pm standard error of the mean (SEM). Statistical significance was accepted for $p<0.05$ as determined by two-way analysis of variance (ANOVA) with Bonferroni post-hoc correction, when comparing variables between male and female mice during the longitudinal assessments. For specific comparisons within the same sex at different ages, a one-way ANOVA was used. Differences in fatty acid composition indices obtained by HR-NMR and in lipid class levels between sexes were assessed using an unpaired Student's $t$-test. Correlations were assessed by the Pearson $r$ coefficient and significance was accepted for $p<0.01$.

\section{3 | RESULTS}

\subsection{Baseline metabolic status}

The baseline assessment of the glycemic response to an oral glucose load, performed at 3 months of age, was similar between both sexes (Figure $1 \mathrm{~A}$, 3 months), but the response to an insulin challenge was different (Figure 1B, 3 months). At 3 months, male mice showed higher blood glucose than females at $3 \mathrm{~h}$ post-ITT (6.7 $\pm 0.6 \mathrm{mM}$ versus $4.5 \pm 0.4 \mathrm{mM}, p<0.01$, Figure $1 \mathrm{G}$ ), but a similar blood glucose drop during the first hour of ITT (Figure 1F) and AAC (Figure 1H). Fasting glucose values were comparable in male and female mice at 3 months (time $=0$ of measurements at 3 months in Figure 1A,B). Sex differences in fasting insulin did not reach statistical significance (Table 2), but QUICKI was lower in male than in female mice ( $p<0.05$, Figure 1C). C-peptide levels were higher in male than in female mice $(p<0.001$, Table 2). Taken together, these observations indicate that, at baseline, male mice are less sensitive than female mice to insulin and display higher insulin secretion in the fasted state, as indicated by $\mathrm{C}$-peptide measurements.

The metabolic evaluation was completed with non-invasive measurements of hepatic lipids by ${ }^{1} \mathrm{H}-\mathrm{MRS}$ in vivo. At baseline, male mice displayed lower HLC than females ( $p<0.01$, Table 2). However, and as expected, body weight at baseline was higher in males than in females (Table 2). The fatty acid composition of cytosolic lipids was characterized with indices derived from the different ${ }^{1} \mathrm{H}$ lipid resonances visible with water suppression (Figure 2A and Table 1). These indices report the average unsaturation and length of the composite hepatic fatty acids, and some differences between male and female mice could be detected at baseline. Namely, the saturation index was smaller in males relative to females $(6.2 \pm 0.2$ versus $7.7 \pm 0.5, p<0.05$, Figure $3 \mathrm{~A}$ ). This index reflects the abundance of methylene protons and depends on both the saturation level and chain length. As indices for the number of double bonds, UFA, PUFA and MUFA were similar between both sexes, the observed difference may be ascribed to shorter fatty acids in male mice (MCL: $14.0 \pm 0.3$ versus $15.7 \pm 0.5, p<0.05$, Figure 3B).

\subsection{Evolution of the metabolic status with age}

With age, body weight increased in both sexes, and male mice at all ages had higher body weight than female mice (Table 2). Blood glucose after a 6-h fast (time $=0$ in graphs in Figure $1 \mathrm{~A}, \mathrm{~B}$ ) did not change with age or sex. Plasma insulin levels did not change with age in either sex, but were higher in male mice throughout the study ( $p<0.001$ for sex with two-way ANOVA). As a result, male mice showed a lower QUICKI than females at all time points assessed (Figure 1C). C-peptide levels did not change with age in either sex, but were always higher in males than in females (Table 2). There were clear changes with age in the glucose curves obtained during OGTT (Figure 1A) and ITT (Figure 1B) in male mice, but not in female mice. These changes are clearly apparent in Figure 1D-H, depicting the longitudinal evolution of OGTT and ITT parameters. Blood glucose at $2 \mathrm{~h}$ post-OGTT (Figure 1D) diverged from similar baseline values and was significantly higher in males than in females at 7 months (7.5 $\pm 0.3 \mathrm{mM}$ versus $10.9 \pm 0.5 \mathrm{mM}, p<0.0001)$ and 11 months $(7.8 \pm 0.3 \mathrm{mM}$ versus $11.3 \pm 0.4 \mathrm{mM}, p<0.0001)$. In female mice, blood glucose at $2 \mathrm{~h}$ post-OGTT decreased with age $(p<0.05)$, whereas no significant changes were noticed in males. The AUCs during OGTT were similar between male and female mice at all ages and did not change with age (Figure 1E). ITT parameters did not change with age in female mice (Figure 1F-H). In males, AAC decreased at 7 and 11 months relative to baseline (Figure $1 \mathrm{H}$ ). This decrease was ascribed to a smaller drop in blood 
(A) 3 Months

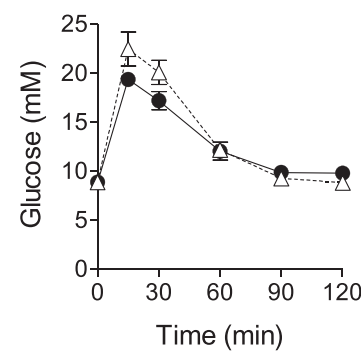

(B)

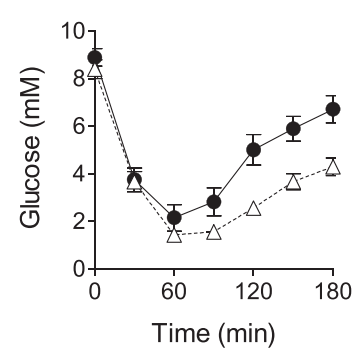

(C)
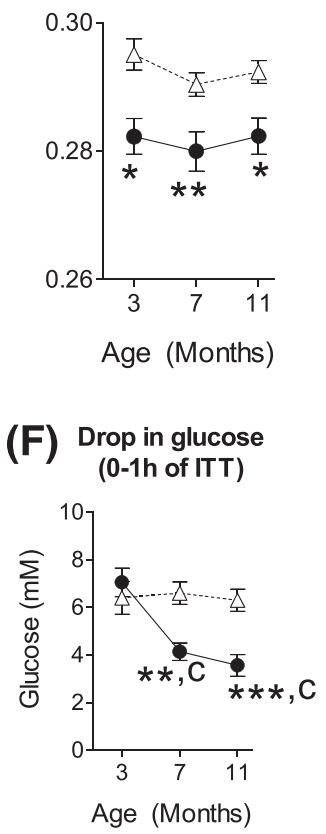

7 Months

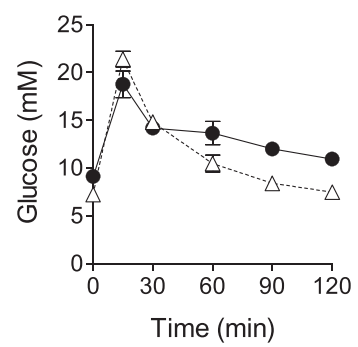

7 Months

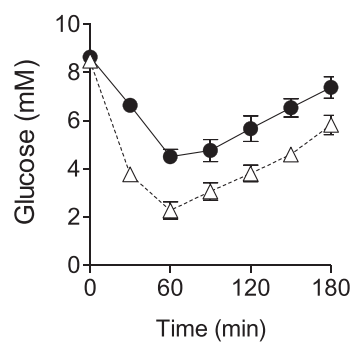

(D) 2h-post OGTT

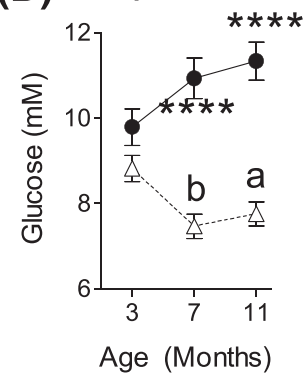

(G)

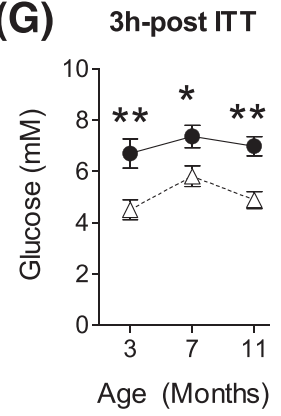

11 Months

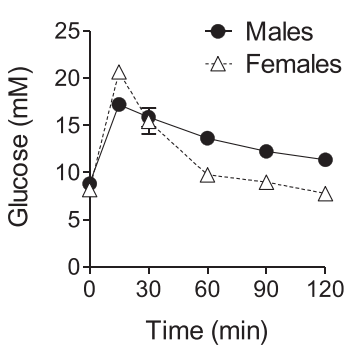

11 Months

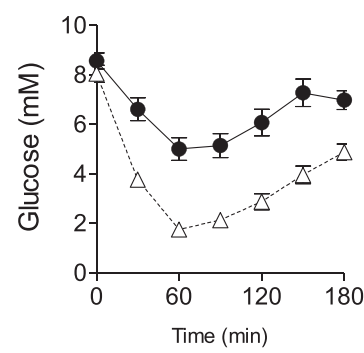

(E) Area Under the Curve (OGTT)

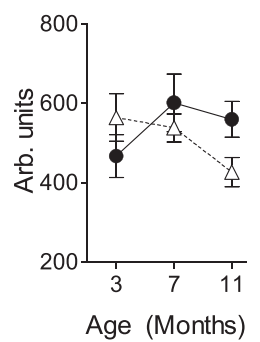

(H) Area Above the Curve (ITT)

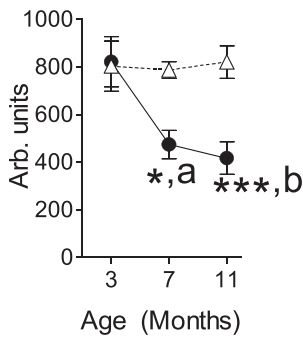

FIGURE 1 Evolution of the metabolic performance of male and female mice. Blood glucose oscillations during the oral glucose tolerance test (OGTT) A and the insulin tolerance test (ITT) B, for three time point assessments: 3, 7 and 11 months. The longitudinal evolution of the quantitative insulin sensitivity check index (QUICKI) C and parameters obtained from the OGTT D, E and ITT F-H, is also shown. Data from male mice are shown as black circles and full lines, and data from female mice are shown as white triangles and dotted lines. ${ }^{*} p<0.05,{ }^{* *} p<0.01,{ }^{* * *} p<$ $0.001,{ }^{* * * *} p<0.0001$ versus females of the same age [two-way analysis of variance (ANOVA) and Bonferroni post-hoc test]. ${ }^{a} p<0.05,{ }^{b} p<0.01$, ${ }^{c} p<0.001$ versus 3 months within the same gender (one-way ANOVA and Bonferroni post-hoc test)

glucose during the first hour of ITT (Figure 1F), as blood glucose at $3 \mathrm{~h}$ post-ITT was similar to that observed at baseline (7.4 $\pm 0.4 \mathrm{mM}$ at $7 \mathrm{months}$ and $6.8 \pm 0.4 \mathrm{mM}$ at 11 months, Figure $1 \mathrm{G}$ ).

HLC increased in male mice from baseline to 7 months without further changes (Table 2). Hence, unlike observations at baseline, no differences in HLC were observed between male and female mice at 7 and 11 months (Table 2). HLC strongly correlated with body weight in male $(r=0.67, p=0.0001$ ), but not female, mice. The saturation index of hepatic cytosolic fatty acids also increased in male mice with age (from $6.2 \pm 0.2$ at baseline to $7.7 \pm 0.3$ at 7 months, $p<0.05$, and $7.5 \pm 0.4$ at 11 months, $p<0.05$, Figure $3 \mathrm{~A}$ ). This change was clearly apparent in the lipid spectral signatures obtained by ${ }^{1} \mathrm{H}$-MRS in vivo from the livers of male mice at the different ages. As depicted in Figure $2 \mathrm{~B}$, the resonance height (main contributor to resonance area) of methylene protons (Lip 1.3) increases relative to that of methyl protons (Lip 0.9), when comparing spectra acquired at 
TABLE 2 Body weight, pancreatic hormones and hepatic lipid content at 3, 7 and 11 months of age in male and female mice

\begin{tabular}{|c|c|c|c|c|c|c|c|c|}
\hline \multirow[b]{2}{*}{ Age (months) } & \multicolumn{2}{|c|}{ Body weight (g) } & \multicolumn{2}{|c|}{ Fasting insulin $(\mu \mathrm{U} / \mathrm{mL})$} & \multicolumn{2}{|c|}{ Fasting C-peptide (ng/mL) } & \multicolumn{2}{|c|}{ Hepatic lipid content (\%) } \\
\hline & Male mice & Female mice & Male mice & Female mice & Male mice & Female mice & Male mice & Female mice \\
\hline 3 & $24.8 \pm 0.4$ & $20.2 \pm 0.3$ & $8.4 \pm 2.8$ & $2.0 \pm 0.2$ & $0.7 \pm 0.1^{b}$ & $0.2 \pm 0.02$ & $1.4 \pm 0.1^{a}$ & $2.7 \pm 0.2$ \\
\hline 11 & $35.0 \pm 0.8$ & $26.0 \pm 0.4$ & $12.2 \pm 4.5$ & $1.8 \pm 0.2$ & $0.9 \pm 0.2^{b}$ & $0.2 \pm 0.02$ & $2.7 \pm 0.3^{d}$ & $2.2 \pm 0.1$ \\
\hline
\end{tabular}

The body weight was always higher in males and increased with age for both sexes $[p<0.0001$, two-way analysis of variance (ANOVA) with Bonferroni posthoc test].

${ }^{a} p<0.01$ and

${ }^{\mathrm{b}} p<0.001$ versus females of the same age (two-way ANOVA with Bonferroni post-hoc test)

${ }^{c} p<0.01$ and

${ }^{\mathrm{d}} p<0.05$ versus 3-month-old males (one-way ANOVA with Bonferroni post-hoc test).

(A)

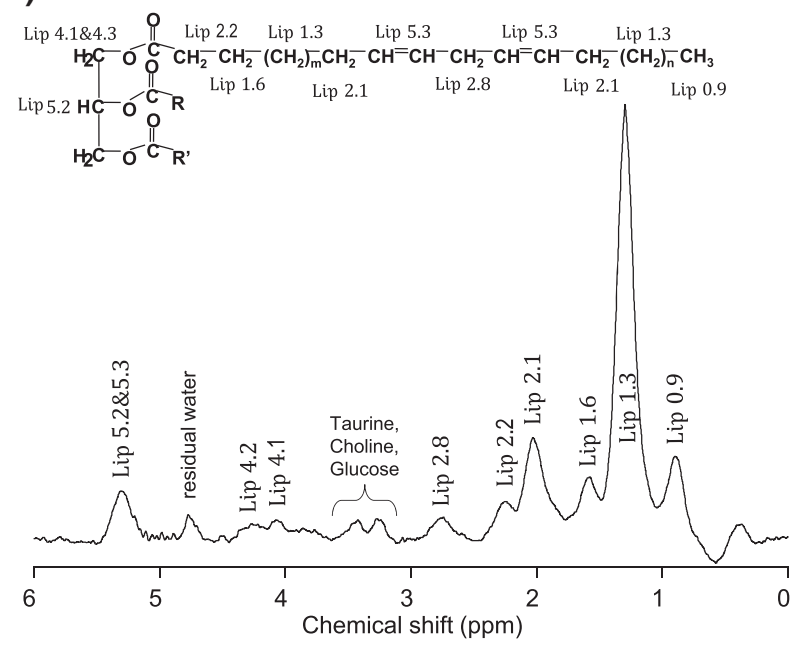

(B)

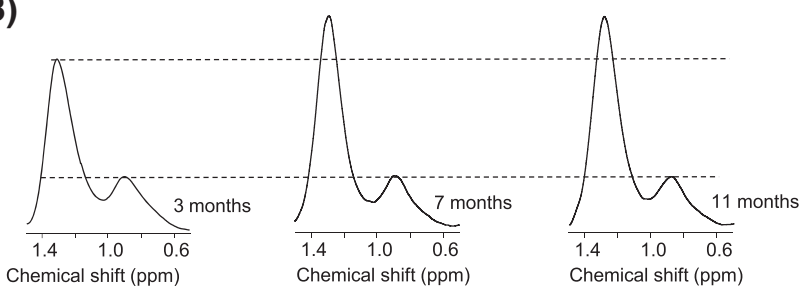

FIGURE 2 Lipid and other metabolite resonances observed in mouse liver by ${ }^{1} \mathrm{H}$-magnetic resonance spectroscopy $\left({ }^{1} \mathrm{H}\right.$-MRS) in vivo. A, The general structure of triglyceride is shown for the identification of the different types of protons in fatty acids, which are labeled according to their chemical shift in the ${ }^{1} \mathrm{H}-\mathrm{MR}$ spectrum acquired with water suppression from the liver of a female mouse at 7 months. B, Alterations with age of the spectral signature observed in male mice in the region from 0.5 to $1.5 \mathrm{ppm}$. The spectra are the sum of those obtained from all male mice at a given age. The broken lines illustrate the increase in the resonance height of Lip 1.3 relative to that of Lip 0.9 (used for normalization to one fatty acid chain) when comparing spectral profiles at 7 and 11 months with that at 3 months

7 and 11 months to those acquired at baseline ( 3 months). The modification of the saturation index with age in males was not accompanied by significant alterations in fatty acid unsaturation indices (double bonds, UFA, PUFA, MUFA, Figure 3C-H) or chain length (Figure 3B). Nevertheless, the combined trends for decreased overall unsaturation (double bonds, UFA) and longer chains seem to explain the increase in the saturation index from baseline to 7 and 11 months.

\section{3 | Associations between metabolic variables}

Correlations between changes in glucose metabolism, increasing body weight and HLC were obtained by combining the datasets from all the longitudinal time points (Table 3). Significant correlations were found between ITT and OGTT parameters and body weight and HLC in male, but not female, mice. There was a small, but significant, positive correlation between body weight and glycemia at $2 \mathrm{~h}$ post-OGTT, and strong negative correlations between body weight and ITT parameters, such as AAC and blood glucose drop during the first hour. However, HLC was 
(A)

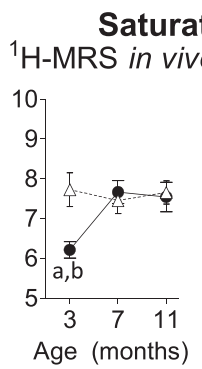

Double Bonds

(C)

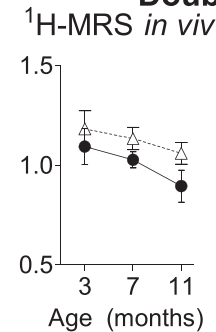

(E)
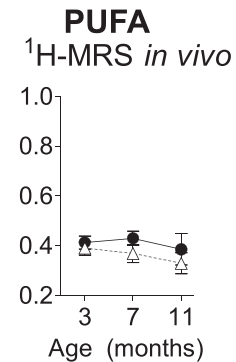

(G)

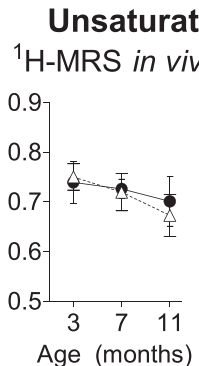

HR-NMR ex vivo

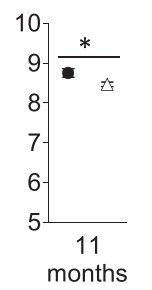

HR-NMR ex vivo

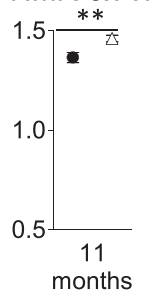

\section{PU Bonds per FA} HR-NMR ex vivo

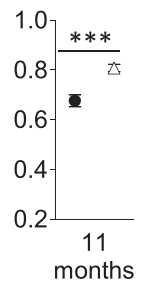

(B)

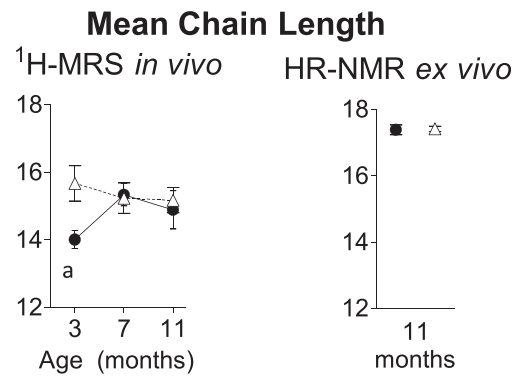

PU Bonds per UFA
(D) ${ }^{1} \mathrm{H}-\mathrm{MRS}$ in vivo HR-NMR ex vivo

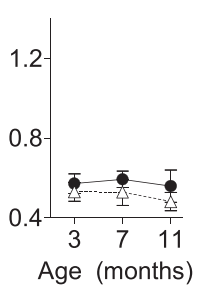

(F)

Saturated Fatty Acids

${ }^{1} \mathrm{H}$-MRS in vivo

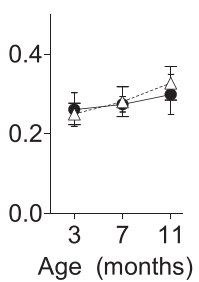

HR-NMR ex vivo

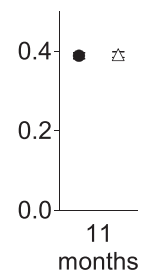

(H) Monounsaturated Fatty Acids

${ }^{1} \mathrm{H}-\mathrm{MRS}$ in vivo

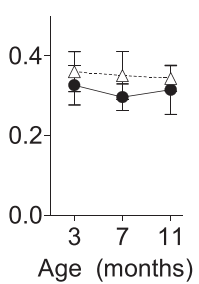

- Males

$\triangle$ Females

FIGURE 3 Indices of fatty acid composition of cytosolic lipids determined longitudinally by ${ }^{1} \mathrm{H}$-magnetic resonance spectroscopy $\left({ }^{1} \mathrm{H}\right.$-MRS) in vivo and of total lipids determined at 11 months by high-resolution, liquid-state, nuclear magnetic resonance (HR-NMR) ex vivo. Data from male mice are shown as black circles and solid lines, and from female mice as white triangles and dotted lines. FA, fatty acid; PU, polyunsaturated; PUFA, polyunsaturated fatty acid; UFA, unsaturated fatty acid. ${ }^{a} p<0.05$ versus females of the same age [two-way analysis of variance (ANOVA) with Bonferroni post-hoc test]. ${ }^{b} p<0.05$ versus 7 - and 11 -month males (one-way ANOVA with Bonferroni post-hoc test). ${ }^{*} p<0.05,{ }^{* *} p<0.01$, ${ }^{* * *} p<0.001$ determined by unpaired Student's t-test

exclusively correlated with ITT parameters. Similar to body weight, there was a negative correlation between HLC and both AAC and blood glucose drop during the first hour. In addition, HLC also showed good correlation with blood glucose at $3 \mathrm{~h}$ post-ITT and with fasting insulin.

\subsection{Endpoint lipidomics of total hepatic lipids}

To complement our longitudinal measurements in vivo, total liver lipids were characterized post-sacrifice, at 11 months of age, by HR-NMR and LC-MS methods. Indices for chain length (Figure 3A,B) and polyunsaturation (Figure 3C-E) obtained from HR-NMR spectra ex vivo were consistently higher than those found by ${ }^{1} \mathrm{H}-\mathrm{MRS}$ in vivo, whereas the UFA index was comparatively lower (Figure 3G). The most abundant membrane phospholipid class, phosphatidylcholine (PtdCho), was clearly identified in the HR-NMR spectra by the resonance of its methyl protons at $3.2 \mathrm{ppm}$ (Figure 4). Bis-allylic protons (Lip 2.8) in diunsaturated fatty acids (DUFA) were resolved from those in chains with a higher degree of polyunsaturation (PUFA), visible at a lower field frequency (Figure 4). In female liver samples, the resonances of olefinic (Lip 5.3) and bis-allylic protons from PUFA were consistently higher than those in male liver samples (Figure 4), translating into a higher average number of double bonds and polyunsaturation indices when comparing the composition of total lipids between the two sexes (Figure 3C-E). Finally, from 
TABLE 3 Correlations between metabolic parameters, body weight and hepatic lipid content in male and female mice during the longitudinal follow-up

\begin{tabular}{|c|c|c|c|c|c|c|c|c|}
\hline & \multicolumn{4}{|c|}{ Male mice } & \multicolumn{4}{|c|}{ Female mice } \\
\hline & \multicolumn{2}{|c|}{ Body weight } & \multicolumn{2}{|c|}{ HLC } & \multicolumn{2}{|c|}{ Body weight } & \multicolumn{2}{|c|}{ HLC } \\
\hline & Pearson $r$ & $\overline{p \text { value }}$ & Pearson $r$ & $p$ value & Pearson $r$ & $\overline{p \text { value }}$ & s & $p$ value \\
\hline AUC & 0.21 & ns & 0.05 & ns & -0.26 & ns & 0.15 & ns \\
\hline Glycemia $_{2 h \text {-post }}$ & 0.49 & $<0.01$ & 0.33 & ns & -0.46 & ns & 0.32 & ns \\
\hline Glycemia drop $_{1 \mathrm{~h} \text {-post }}$ & -0.68 & $<0.001$ & -0.57 & $<0.01$ & -0.02 & ns & 0.22 & ns \\
\hline Glycemia $_{3 h-p o s t}$ & 0.20 & ns & 0.55 & $<0.01$ & -0.25 & ns & 0.33 & ns \\
\hline \multicolumn{9}{|l|}{ 6-h fasting parameters } \\
\hline Glucose & 0.83 & ns & 0.47 & ns & -0.17 & ns & 0.2691 & ns \\
\hline
\end{tabular}

AAC, area above the curve; AUC, area under the curve; HLC, hepatic lipid content; ITT, insulin tolerance test; OGTT, oral glucose tolerance test; QUICKI, quantitative insulin sensitivity check index.

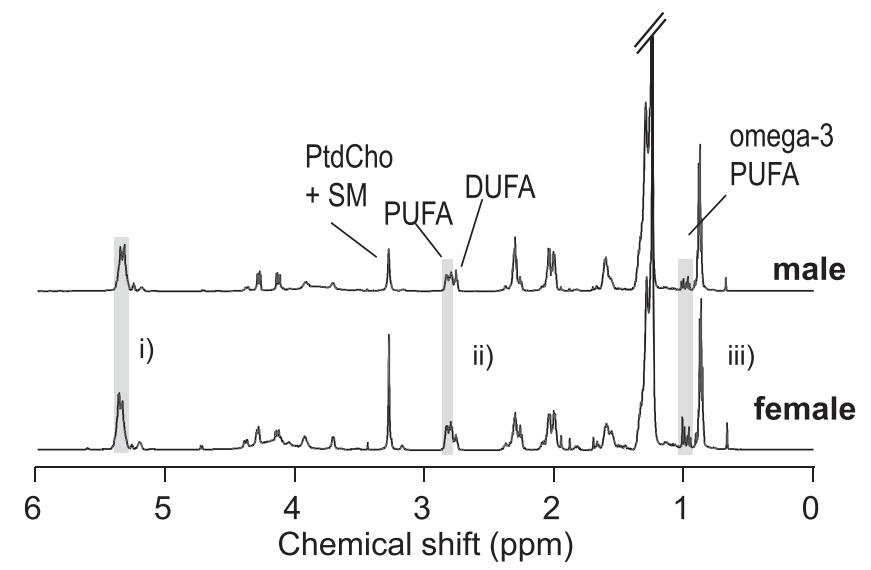

FIGURE 4 Representative high-resolution, liquid-state, nuclear magnetic resonance (HR-NMR) spectra of total lipids extracted from the liver of one male and one female mouse. Spectra are scaled to the resonance of the terminal methyl group (Lip 0.9) and spectral differences are shaded in gray. For identification of the lipid resonances in the fatty acid chain, see Figure 2. Distinct spectral differences correspond to higher numbers of double bonds (i), polyunsaturated double bonds per fatty acyl chain and unsaturated fatty acyl chain (ii) and omega-3 PUFA (iii). DUFA, diunsaturated fatty acid; PtdCho, phosphatidylcholine; PUFA, polyunsaturated fatty acid; SM, sphingomyelin

HR-NMR spectra, we estimate that omega-3 PUFA represented $12 \pm 0.6 \%$ of the fatty acids in total lipids in females and a significantly lower proportion, $10 \pm 0.5 \%(p<0.01)$, in males.

To obtain detailed insight into lipid classes and species distribution profiles, we performed LC-MS for lipid separation, identification and relative quantification (Figure 5). The abundance of certain lipid classes exhibited sexual dimorphism, whereas others occurred in similar amounts in both sexes (Figure 5A). In particular, the levels of triglycerides (TG), the principal cytosolic lipids, were similar between male and female liver samples. The levels of diacylglycerols in female liver samples were about one-half of those found in males. No differences were found in the levels of the principal class of membrane phospholipids (PtdCho), but the abundance of phosphatidylinositols (Ptdlns) and phosphatidylethanolamines (PtdEt) was higher in female than in male livers, whereas phosphatidylglycerols (PG) were lower in female than in male liver samples. We also found differences in a less prominent class, lyso-bis-phosphatidic acid (LBPA), whose levels were increased in the livers of female mice relative to males. We further investigated the species distribution profile of major lipid classes, i.e. TG and the principal membrane phospholipids PtdCho, PtdEt, Ptdlns and sphingomyelins (SM). In TG, species 54:4 existed in a larger proportion in liver lipids from female, relative to male, mice, which, instead, showed a larger proportion of species 52:4 (Figure 5B). More pronounced differences in the species distribution profile were found in membrane phospholipids. PtdCho (Figure 5C) from female livers showed a higher proportion of very long chain and highly polyunsaturated species (38:4; 38:5, 38:6, 40:6) than that found in PtdCho from male livers. Conversely, male livers exhibited a higher proportion of shorter PtdCho with up to three double bonds (34:1; 34:2, 36:3). A greater abundance of highly unsaturated species was also observed in PtdEt (Figure 5D) from female liver 

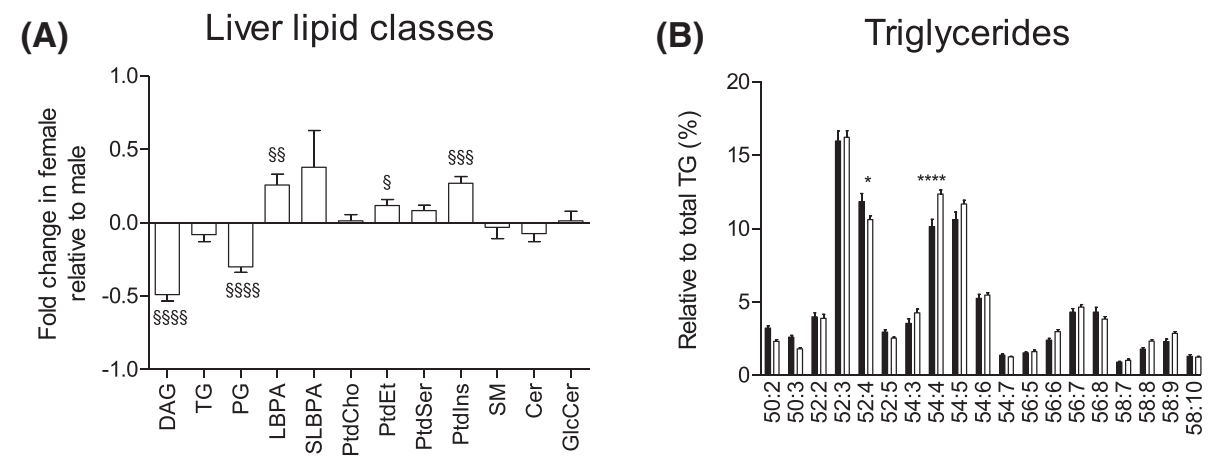

(C)

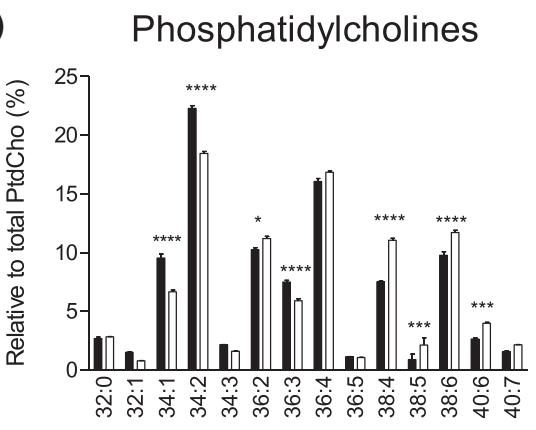

(D) Phosphatidylethanolamines

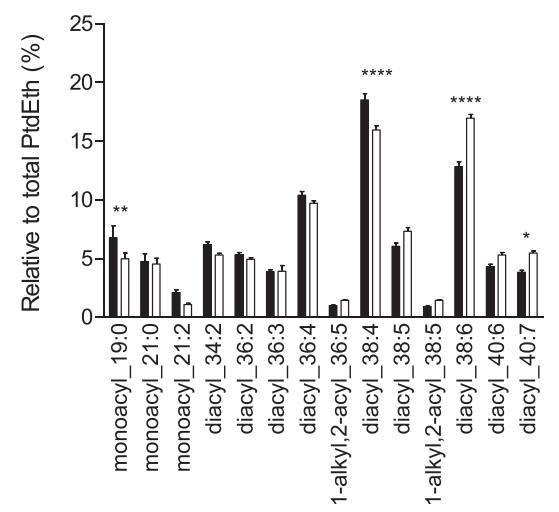

(E)
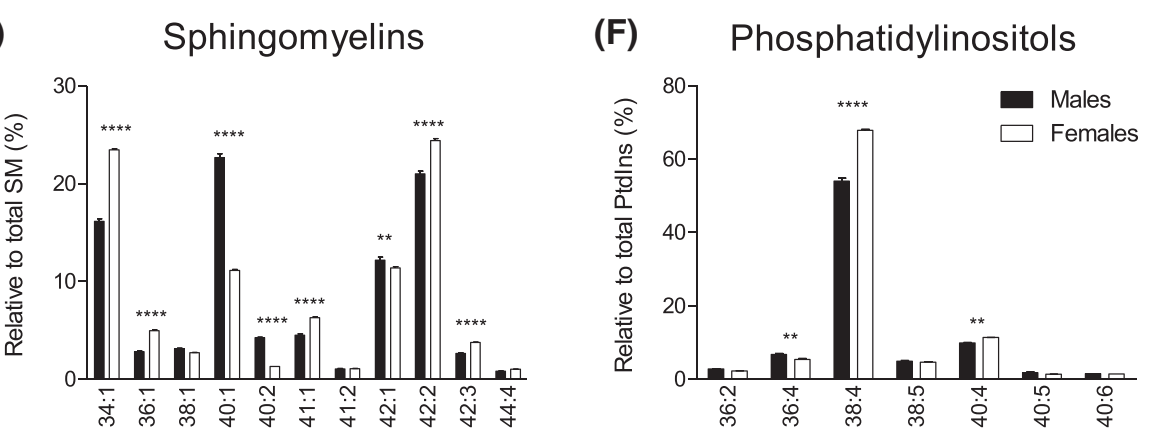

FIGURE 5 Sexual dimorphism in liver lipid classes A, and species B-F, assessed by liquid chromatography-mass spectrometry (LC-MS). When comparing lipid classes between genders A, significant differences from zero, with zero denoting no change, are marked for ${ }^{\S} p<0.05$, ${ }^{\S \S} p<$ $0.01,{ }^{\S \S} P<0.001$ and ${ }^{\S \S \S \S} p<0.0001$. The percentage contribution of lipid species to the total class is shown in B-F, in male (black bars) and female (white bars) liver samples, and differences between genders are marked for ${ }^{*} p<0.05,{ }^{* *} p<0.01,{ }^{* * *} p<0.001$ and ${ }^{* * * *} p<0.0001$ [two-way analysis of variance (ANOVA) and Bonferroni multiple comparison post-hoc test]. Unless specified otherwise, lipid species are tri- or di-acylated molecules. Cer, ceramides; DAG, diacylglycerols; GlcCer, glucosylceramides; LBPA, lyso-bis-phosphatidic acid; PG, glycerophosphoglycerols; PtdCho, phosphatidylcholines; PtdEt, phosphatidylethanolamines; Ptdlns, phosphatidylinositols; PtdSer, Phosphatidylserines; SLBPA, semi-lyso-bisphosphatidic acid; SM, sphingomyelins; TG, triglycerides

samples, with a larger proportion of 38:6 and 40:7 than that observed in male livers. SM (Figure 5E) also exhibited strong sexual dimorphism in the species distribution profile, with females showing a higher contribution from shorter monounsaturated species 34:1 and 36:1, but also from longer polyunsaturated species 42:2 and 42:3. However, the most abundant SM species in males was 40:1, reaching a proportion that was twice that found in females. Other SM species more abundant in males were 40:2 and 42:1. The principal Ptdlns (Figure 5F) species 38:4 showed a higher relative content in female than in male liver samples.

Finally, we investigated the association between the most prominent changes in total liver lipids and the metabolic parameters of the corresponding mice at the age of 11 months (Figure 6). In female mice, the percentage contribution of omega-3 PUFA was strongly correlated with insulin sensitivity assessed by QUICKI (Figure 6A, $r=0.78, p<0.01$ ). Except for this association, no other significant correlation was found between metabolic performance and total lipid components in a sex-specific manner. However, several correlations were present when analyzing male and female mice together, probably as a result of a sex-related clustering effect. In brief, insulin sensitivity (QUICKI and ITT parameters) increased with omega-3 PUFA (Figure 6A,B) and PtIns (Figure 6C-E), which were both higher in female liver samples (Figure 5A), and decreased with lipid classes more prominent in males, i.e. PG (Figure 6F) and diacylglycerols (Figure 6G). Glucose tolerance declined with increasing PG levels (Figure 6H, I), higher in male liver samples. 
(A)

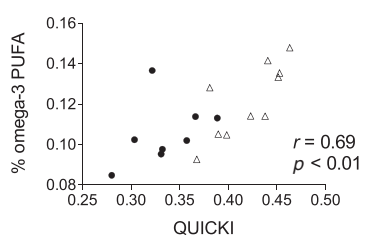

(D)

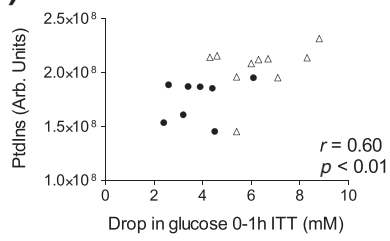

(G)

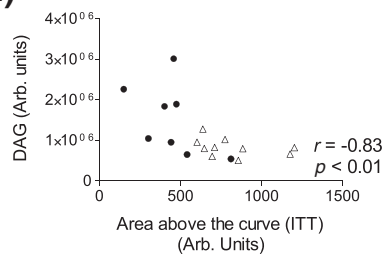

(B)

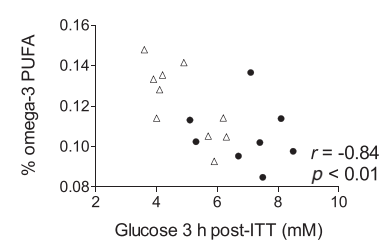

(E)

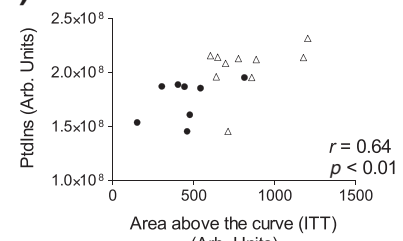

(H)

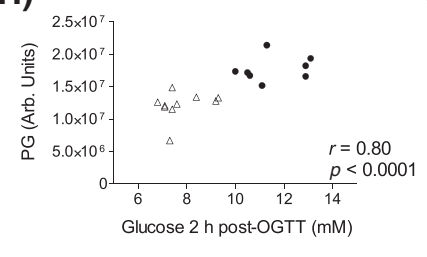

(C)

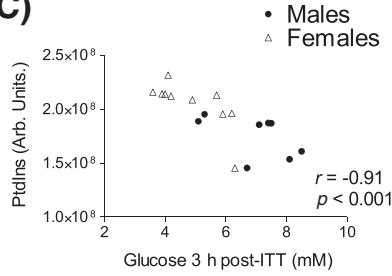

(F)

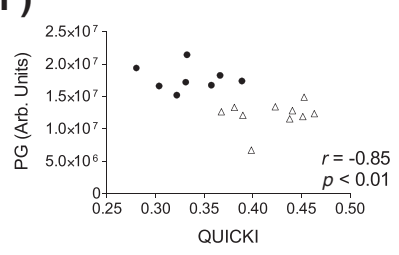

(I)

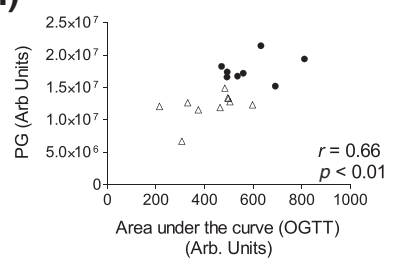

FIGURE 6 Significant correlations between total lipid components exhibiting sexual dimorphism and metabolic scores. Metabolic performance was characterized from the quantitative insulin sensitivity check index (QUICKI), blood glucose at $3 \mathrm{~h}$ post-ITT, area above the curve for blood glucose oscillations during ITT, blood glucose drop during the first hour of ITT, blood glucose at $2 \mathrm{~h}$ post-OGTT and area under the curve for blood glucose oscillations during OGTT. Males are represented with black circles and females with white triangles. DAG, diacylglycerols; ITT, insulin tolerance test; OGTT, oral glucose tolerance test; PG, glycerophosphoglycerols; Ptdlns, phosphatidylinositols; PUFA, polyunsaturated fatty acids

\section{4 | DISCUSSION}

We followed glucose homeostasis and hepatic lipids in aging male and female mice housed under standard conditions with free access to a regular chow diet. We observed age-related changes in glucose homeostasis in male mice, which correlated with weight gain and HLC. As expected, the metabolic performance diverged significantly between male and female mice, but the sexually dimorphic response to glucose and insulin challenges was not accompanied by sex differences in HLC or fatty acid composition, as monitored by ${ }^{1} \mathrm{H}-\mathrm{MRS}$ in vivo. Instead, further lipid profiling by HR-NMR and LC-MS revealed striking sexual dimorphism in lipid membrane species, supporting a link between biologically active lipid intermediates, not necessarily stored TG, and susceptibility to metabolic dysregulation in aged mice.

We used different approaches to assess hepatic lipids, and each method has particular strengths and limitations. Non-invasive ${ }^{1} \mathrm{H}-\mathrm{MRS}$ in vivo is highly suited to longitudinal measurements and exclusively detects mobile lipids in the cytosol. Conversely, both HR-NMR and LC-MS additionally detect the membrane lipids dissolved in the organic medium during the extraction procedure. Relative to HR-NMR, LC-MS is more sensitive for the detection of less abundant species and is better able to resolve the various classes and species in the total lipid mixture. The fatty acids observed by ${ }^{1} \mathrm{H}$-MRS in vivo are mainly constituents of TG, which account for over $95 \%$ of the lipid content in cytosolic lipid droplets from murine hepatocytes. ${ }^{29}{ }^{1} \mathrm{H}$-MRS in vivo indicated similar HLC between male and female mice at 11 months of age (Table 2), in agreement with the similar TG content determined by LC-MS in the respective liver tissue extracts (Figure 5A). LC-MS and lipidomics analysis reported modest differences in TG species between the two sexes. Such differences were not apparent in the indices reporting the averaged composition of hepatic fatty acids estimated from ${ }^{1} \mathrm{H}$-MR spectra acquired in vivo, which were similar between male and female mice at 11 months of age (Figure 3 , ${ }^{1} \mathrm{H}$-MRS in vivo graphs). In rodent models of hepatic steatosis ${ }^{18,19,30}$ and diabetes, ${ }^{21}$ differences in fatty acid composition could be detected by ${ }^{1} \mathrm{H}-\mathrm{MRS}$ in vivo, supporting the value of this technique in monitoring the remodeling of cytosolic fatty acids within the scope of severe metabolic impairments.

Compared with the indices obtained by ${ }^{1} \mathrm{H}-\mathrm{MRS}$ in vivo, HR-NMR ex vivo reported a higher number of double bonds and degree of polyunsaturation, and lower UFA, reproducing our previous observations ${ }^{21}$ and indicating the presence of membrane-derived fatty acids that are not detected by ${ }^{1} \mathrm{H}-\mathrm{MRS}$ in vivo. We found distinct HR-NMR spectral signatures between male and female total lipid samples (Figure 4). and therefore differences in several fatty acid composition indices (Figure 3, HR-NMR ex vivo graphs). As $\mathrm{HLC}$ and indices determined by ${ }^{1} \mathrm{H}-\mathrm{MRS}$ in vivo were similar between the sexes at 11 months, these differences indicate sexual dimorphism in the fatty acid composition of membrane lipids. Indeed, lipidomics after LC-MS separation and identification showed that the principal membrane lipids, PtdCho and PtdEt, were characterized by a greater contribution of highly polyunsaturated lipid species in the livers of female mice, relative to males (Figure 5C,D), similar to findings from other authors. ${ }^{31-33}$ These lipid data provide an explanation for the higher average number of double bonds and polyunsaturated bonds, estimated from HR-NMR spectra, in female liver samples, when comparing with males. 
To investigate the association between liver lipids and glucose homeostasis, we also monitored changes in blood glucose in response to glucose and insulin challenges. In both cases, the decrease in blood glucose is driven by insulin-dependent stimulation of peripheral glucose uptake and inhibition of hepatic glucose production. Glucose production following a glucose load is suppressed after a 20 -min delay, ${ }^{34}$ but this pathway still contributes one-third to plasma glucose in the last hour of OGTT. ${ }^{35,36}$ Therefore, adjustments of hepatic glucose production in response to insulin are mostly reflected in the last time points of the metabolic tests. In male mice, we found excellent correlations between HLC and parameters from ITT, a test that enables a direct assessment of basal insulin sensitivity. ${ }^{37}$ Of note, blood glucose at $3 \mathrm{~h}$ post-ITT showed good correlation with HLC independent of body weight (Table 3). Such specificity supports a close relation between HLC and insulin-mediated adjustments of glucose production at the end of the test. HLC also correlated with fasting insulin levels, which reflect insulin sensitivity. ${ }^{38}$ No correlation was found between HLC and fasting C-peptide concentrations in the blood of male mice, indicating that insulin secretion was not compromised with increasing HLC. A study using a short-term high-sucrose diet also failed to find evidence of perturbed insulin secretion, despite the presence of hepatic steatosis and fasting hyperinsulinemia in rats. ${ }^{39}$ Therefore, at least in this study and ours, a less efficient hepatic insulin extraction appears to be a common mechanism that links increased HLC to peripheral hyperinsulinemia. It should be noted that HLC observed in our study is within the normal range ${ }^{18,21}$ and below the $5-20 \%$ range found in models of liver steatosis. ${ }^{18,19,40}$ As increased hepatic insulin exposure is known to contribute to the metabolic dysregulation in chronic steatosis, ${ }^{41}$ the relationship between HLC and hepatic insulin extraction may depend on the extent of hepatic steatosis. In contrast with ITT, OGTT explores the action of endogenous insulin secreted by the pancreas. ${ }^{38,42}$ Although HLC and OGTT parameters have been reported to correlate in experimental liver steatosis, ${ }^{35}$ we did not find a correlation in the current study. Instead, OGTT performance changed over time in male mice in correlation with their body weight. Although we measured fasting insulin and C-peptide concentrations, the lack of information on the excursions of these pancreatic hormones during OGTT limits our ability to assess changes in glucose-stimulated insulin secretion. Perturbed $\beta$-cell function could explain, at least in part, aging-induced glucose intolerance in male mice. In support of this notion, aged mice show a comparative lack of $\beta$-cell plasticity to adapt to metabolic insults, such as high-fat diet feeding. ${ }^{43}$ Both insulin action and secretion after the ingestion of a mixed meal decrease with age in men, but not in women. ${ }^{44}$ However, both men and women show increased hepatic insulin extraction with age, ${ }^{44}$ and this would also reduce peripheral insulin exposure, thereby influencing the dynamics of the glycemic response to the glucose challenge.

Although, in male mice, HLC strongly correlated with body weight, this correlation was absent in females (Table 3). In ad libitum-fed rats, increased body weight with age is associated with the expansion of visceral fat mass, ${ }^{5}$ and visceral fat expansion contributes directly to hepatic lipid accumulation in obese mice. ${ }^{45}$ Therefore, visceral fat possibly mediates the increase in HLC associated with body weight in male mice in our study. In contrast, the lack of hepatic lipid accumulation in female mice, which nonetheless displayed increasing body weight, could relate to a better capacity for estrogen-promoted subcutaneous fat storage. ${ }^{46}$ Indeed, experimentally induced estrogen deficiency results in increased visceral fat mass ${ }^{47}$ and ectopic lipid deposition in the liver. ${ }^{48}$ By their action on hepatic lipid metabolism, estrogens are also known modulators of insulin sensitivity. ${ }^{49}$ Interestingly, under normal conditions. hepatic lipid levels are comparable between both sexes, or even higher in females, as observed in this and other studies. ${ }^{50,51}$ In addition, the association of insulin sensitivity with HLC was absent in females, which showed better scores than males with the same hepatic lipid load, i.e. at the older ages of 7 and 11 months (Table 2 and Figure 1). Therefore, the amount of stored lipids in the liver, reported by both ${ }^{1} \mathrm{H}-\mathrm{MRS}$ in vivo and LC-MS as TG, appears to be unrelated to the sexual dimorphism in metabolic health.

As demonstrated by studies with genetically modified mice accumulating TG in the liver, hepatic steatosis may be dissociated from insulin resistance. ${ }^{52}$ Rather than hepatic TG per se, lipotoxic non-TG lipids contribute to metabolic disease. ${ }^{53-55}$ In correlation with metabolic scores, we found that several non-TG lipids with biological activity exhibited sexual dimorphism in aged mice. Diacylglycerols are implicated in insulin resistance by their ability to perturb the insulin signaling cascade via protein kinase $C(P K C) .{ }^{56,57}$ Diacylglycerols are intermediate species in the biosynthesis of TG, but may also be generated from membrane phospholipids. Membrane Ptdlns, in particular, has been suggested as a relevant source of diacylglycerols for PKC activation. ${ }^{58,59}$ At 11 months, male mice showed higher hepatic levels of diacylglycerols than female mice and, conversely, lower levels of Ptdlns. Indeed, there was a negative correlation between the levels of these two classes when considering male and female mice together $(r=-0.68, p<0.001)$. The release of diacylglycerols from membrane Ptdlns in males, with the effect on PKC signaling, could explain their lower insulin sensitivity relative to females. Sexual dimorphism in diacylglycerols and Ptdlns was strongly correlated with insulin sensitivity reported by ITT parameters (Figure 6B,C,F and G). These findings are in good agreement with a study showing that estradiol administration to ovariectomized mice strongly reduced hepatic diacylglycerol levels and PKC activation. ${ }^{60}$

In agreement with other studies, ${ }^{28,61}$ the great majority of Ptdlns were C38:4 species, i.e. with one C18:0 (stearoyl) and one C20:4 (arachidonyl) chain. Ptdlns is selectively enriched with arachidonic acid via recycling of Ptdlns-derived diacylglycerols and chain remodeling. ${ }^{62,63}$ Because arachidonic acid is a precursor to pro-inflammatory eicosanoids, ${ }^{62,64}$ its incorporation in membrane Ptdlns reduces intracellular inflammation by controlling arachidonic acid levels. Accordingly, the maintenance of metabolic control in female mice is indeed associated with the absence of inflammatory liver damage in the face of hepatic lipid accumulation. ${ }^{15}$ In addition, female rats have been shown to display a higher capacity to synthesize the anti-inflammatory omega-3 PUFA, docosahexanoic acid, ${ }^{33,65}$ a mechanism mediated by estrogens. ${ }^{66}$ In line with these reports, we detected higher amounts of omega-3 PUFA in total liver lipids of female than male mice. Given the association of metabolic disease with inflammation, 6,64 a higher pro-inflammatory tonus in males could also account for the sex-specific changes in metabolic health in our study. Levels of omega-3 PUFA were indeed strongly correlated with metabolic health parameters in our experimental paradigm (Figure 6D,E). 


\section{5 | CONCLUSIONS}

This study shows that decreasing insulin sensitivity evolves in association with increasing HLC in male mice housed under standard conditions, but not in females. This finding was not accompanied by marked sex-related differences in the fatty acid composition of the major cytosolic lipids, as determined by both ${ }^{1} \mathrm{H}$-MRS in vivo and LC-MS. We found strong sexual dimorphism in diacylglycerol, Ptdlns and omega-3 PUFA content in aged mice. Rather than stored TG, it is these bioactive lipids, whose levels are modulated by estrogen activity, ${ }^{60,66}$ that influence the evolution of the metabolic performance of mice housed under standard conditions. In males, increases in the hepatic cytosolic lipid load without proportional compensation from bioactive lipids probably explains the association of insulin sensitivity with HLC.

\section{ACKNOWLEDGEMENTS}

The authors are grateful to Dr João M. N. Duarte for his encouragement and constructive comments during the preparation of the manuscript, to Dr Hikari Yoshihara for thorough proof-reading and editing of the manuscript, and to Dr Carola Romero and Ms Anne-Catherine Clerc for their expert technical support in performing the metabolic tests. The authors also acknowledge the financial support from the Center for Biomedical Imaging (CIBM) of the University of Lausanne (UNIL), University of Geneva (UNIGE), Geneva University Hospital (HUG), Lausanne University Hospital (CHUV), Swiss Federal Institute of Technology (EPFL) and the Leenaards and Louis-Jeantet Foundations.

\section{REFERENCES}

1. Seals DR, Bell C. Chronic sympathetic activation: consequence and cause of age-associated obesity? Diabetes. 2004;53:276-284.

2. Matsuzawa Y, Shimomura I, Nakamura T, Keno Y, Kotani K, Tokunaga K. Pathophysiology and pathogenesis of visceral fat obesity. Obes Res. 1995;3:187s-194s.

3. Huffman DM, Barzilai N. Role of visceral adipose tissue in aging. Biochim Biophys Acta. 1790;2009:1117-1123.

4. White UA, Tchoukalova YD. Sex dimorphism and depot differences in adipose tissue function. Biochim Biophys Acta. 1842;2014:377-392.

5. Barzilai N, Banerjee S, Hawkins M, Chen W, Rossetti L. Caloric restriction reverses hepatic insulin resistance in aging rats by decreasing visceral fat. $J$ Clin Invest. 1998;101:1353-1361.

6. Hocking S, Stewart R, Brandon A, et al. Subcutaneous fat transplantation alleviates diet-induced glucose intolerance and inflammation in mice. Diabetologia. 2015;58:1587-1600.

7. Foster MT, Shi H, Seeley RJ, Woods SC. Removal of intra-abdominal visceral adipose tissue improves glucose tolerance in rats: role of hepatic triglyceride storage. Physiol Behav. 2011;104:845-854.

8. Samuel VT, Liu Z-X, Qu X, et al. Mechanism of hepatic insulin resistance in non-alcoholic fatty liver disease. J Biol Chem. 2004;279:32345-32353.

9. Stefan N, Häring H-U. The metabolically benign and malignant fatty liver. Diabetes. 2011;60:2011-2017.

10. Viljanen APM, lozzo P, Borra R, et al. Effect of weight loss on liver free fatty acid uptake and hepatic insulin resistance. J Clin Endocrinol Metab. 2009;94:50-55.

11. Pan J-J, Fallon MB. Gender and racial differences in nonalcoholic fatty liver disease. World J Hepatol. 2014;6:274-283.

12. Pramfalk C, Pavlides M, Banerjee R, et al. Sex-specific differences in hepatic fat oxidation and synthesis may explain the higher propensity for NAFLD in men. J Clin Endocrinol Metab. 2015;100:4425-4433.

13. Comhair T, Garcia Caraballo S, Dejong C, Lamers W, Kohler SE. Dietary cholesterol, female gender and n-3 fatty acid deficiency are more important factors in the development of non-alcoholic fatty liver disease than the saturation index of the fat. Nutr Metab (Lond). 2011;8:4.

14. Senthil Kumar SPD, Shen M, Spicer EG, et al. Distinct metabolic effects following short-term exposure of different high-fat diets in male and female mice. Endocr J. 2014;61:457-470.

15. Ganz M, Csak T, Szabo G. High fat diet feeding results in gender specific steatohepatitis and inflammasome activation. World J Gastroenterol. 2014;20:8525-8534.

16. Christensen KE, Wu Q, Wang X, Deng L, Caudill MA, Rozen R. Steatosis in mice is associated with gender, folate intake, and expression of genes of onecarbon metabolism. J Nutr. 2010;140:1736-1741.

17. Das M, Gabriely I, Barzilai N. Caloric restriction, body fat and ageing in experimental models. Obes Rev. 2004;5:13-19.

18. Ramamonjisoa N, Ratiney H, Mutel E, et al. In vivo hepatic lipid quantification using MRS at 7T in a mouse model of glycogen storage disease type 1a. J Lipid Res 2013;54:2010-2022.

19. van Werven JR, Marsman HA, Nederveen AJ, ten Kate FJ, van Gulik TM, Stoker J. Hepatic lipid composition analysis using 3.0-T MR spectroscopy in a steatotic rat model. Magn Reson Med. 2012;30:112-121.

20. Ye Q, Danzer CF, Fuchs A, Vats D, Wolfrum C, Rudin M. Longitudinal evaluation of hepatic lipid deposition and composition in ob/ob and ob/+ contro mice. NMR Biomed. 2013;26:1079-1088.

21. Soares AF, Lei H, Gruetter R. Characterization of hepatic fatty acids in mice with reduced liver fat by ultra-short echo time ${ }^{1} \mathrm{H}$-MRS at $14.1 \mathrm{~T}$ in vivo. NMR Biomed. 2015;28:1009-1020.

22. Gruetter R, Tkáč I. Field mapping without reference scan using asymmetric echo-planar techniques. Magn Reson Med. 2000;43:319-323.

23. Tkáč I, Starčuk Z, Choi IY, Gruetter R. In vivo ${ }^{1} \mathrm{H}$ NMR spectroscopy of rat brain at 1 ms echo time. Magn Reson Med. 1999;41:649-656.

24. Provencher SW. Automatic quantitation of localized in vivo ${ }^{1} \mathrm{H}$ spectra with LCModel. NMR Biomed. 2001;14:260-264.

25. Folch J, Lees M, Stanley GHS. A simple method for the isolation of total lipids from animal tissues. J Biol Chem. 1957;226:497-509.

26. Duarte JAG, Carvalho F, Pearson M, et al. A HFD suppresses de novo lipogenesis and desaturation, but not elongation and triglyceride synthesis in mice. J Lipid Res 2014;55:2541-2553. 
27. Hartler J, Trötzmüller M, Chitraju C, Spener F, Köfeler HC, Thallinger GG. Lipid data analyzer: unattended identification and quantitation of lipids in LC-MS data. Bioinformatics. 2011;27:572-577.

28. Rouzer CA, Ivanova PT, Byrne MO, Milne SB, Marnett L, Brown HA. Lipid profiling reveals arachidonate deficiency in RAW264.7 cells: structural and functional implications. Biochemistry. 2006;45:14795-14808.

29. Fauland $\mathrm{A}$, Köfeler $\mathrm{H}$, Trötzmüller $\mathrm{M}$, et al. A comprehensive method for lipid profiling by liquid chromatography-ion cyclotron resonance mass spectrometry. J Lipid Res. 2011;52:2314-2322.

30. Ye Q, Danzer C, Fuchs A, Wolfrum C, Rudin M. Hepatic lipid composition differs between ob/ob and ob/+ control mice as determined by using in vivo localized proton magnetic resonance spectroscopy. Magn Reson Mater Phys Biol Med. 2012;25:381-389.

31. Marks K, Kitson A, Stark K. Hepatic and plasma sex differences in saturated and monounsaturated fatty acids are associated with differences in expression of elongase 6, but not stearoyl-CoA desaturase in Sprague-Dawley rats. Genes Nutr. 2013;8:317-327.

32. Burdge GC, Slater-Jefferies JL, Grant RA, et al. Sex, but not maternal protein or folic acid intake, determines the fatty acid composition of hepatic phospholipids, but not of triacylglycerol, in adult rats. Prostaglandins Leukot Essent Fatty Acids. 2008;78:73-79.

33. Kitson AP, Smith TL, Marks KA, Stark KD. Tissue-specific sex differences in docosahexaenoic acid and $\Delta 6$-desaturase in rats fed a standard chow diet. Appl Physiol Nutr Metab. 2012;37:1200-1211.

34. Smadja C, Morin J, Ferré PJG. Metabolic fate of a gastric glucose load in unrestrained rats bearing a portal vein catheter. Am J Physiol. 1988;254: E407-E413.

35. Delgado TC, Barosa C, Nunes PM, Cerdán S, Geraldes CFGC, Jones JG. Resolving the sources of plasma glucose excursions following a glucose tolerance test in the rat with deuterated water and [U- $\left.{ }^{13} \mathrm{C}\right]$ glucose. PLoS One. 2012;7: e34042.

36. Nunes PM, Jarak I, Heerschap A, Jones JG. Resolving futile glucose cycling and glycogenolytic contributions to plasma glucose levels following a glucose load. Magn Reson Med. 2014;71:1368-1373.

37. Heikkinen S, Argmann CA, Champy M-F, Auwerx J. Evaluation of glucose homeostasis. Curr Protoc Mol Biol. 2007;29B.3.1-29B.3.22.

38. Stumvoll M, Mitrakou A, Pimenta W, et al. Use of the oral glucose tolerance test to assess insulin release and insulin sensitivity. Diabetes Care. 2000;23:295-301.

39. Martins FO, Delgado TC, Viegas J, et al. Mechanisms by which the thiazolidinedione troglitazone protects against sucrose-induced hepatic fat accumulation and hyperinsulinaemia. Br J Pharmacol. 2016;173:267-278.

40. Nunes PM, Wright AJ, Veltien A, et al. Dietary lipids do not contribute to the higher hepatic triglyceride levels of fructose- compared to glucose-fed mice. FASEB J. 2014;28:1988-1997.

41. Finucane FM, Sharp SJ, Hatunic M, et al. Liver fat accumulation is associated with reduced hepatic insulin extraction and beta cell dysfunction in healthy older individuals. Diabetol Metab Syndr. 2014;6:43-43.

42. Andrikopoulos S, Blair AR, Deluca N, Fam BC, Proietto J. Evaluating the glucose tolerance test in mice. Am J Physiol Endocrinol Metab. 2008;295: E1323-E1332.

43. Tschen S-I, Dhawan S, Gurlo T, Bhushan A. Age-dependent decline in $\beta$-cell proliferation restricts the capacity of $\beta$-cell regeneration in mice. Diabetes. 2009;58:1312-1320.

44. Basu R, Dalla Man C, Campioni M, et al. Effects of age and sex on postprandial glucose metabolism. Diabetes. 2006;55:2001

45. Foster MT, Softic S, Caldwell J, Kohli R, deKloet AD, Seeley RJ. Subcutaneous adipose tissue transplantation in diet-induced obese mice attenuates metabolic dysregulation while removal exacerbates it. Physiol Rep. 2013;1:e00015.

46. Davis KED, Neinast M, Sun K, et al. The sexually dimorphic role of adipose and adipocyte estrogen receptors in modulating adipose tissue expansion, inflammation, and fibrosis. Mol Metab. 2013;2:227-242.

47. Litwak SA, Wilson JL, Chen W, et al. Estradiol prevents fat accumulation and overcomes leptin resistance in female high-fat diet mice. Endocrinology. 2014;155:4447-4460.

48. Paquette A, Shinoda M, Lhoret RR, Prud'homme D, Lavoie J-M. Time course of liver lipid infiltration in ovariectomized rats: impact of a high-fat diet. Maturitas. 2007;58:182-190.

49. Bryzgalova G, Gao H, Ahren B, et al. Evidence that oestrogen receptor-a plays an important role in the regulation of glucose homeostasis in mice: insulin sensitivity in the liver. Diabetologia. 2006;49:588-597.

50. Atshaves BP, Payne HR, McIntosh AL, et al. Sexually dimorphic metabolism of branched-chain lipids in C57BL/6J mice. J Lipid Res. 2004;45:812-830.

51. Yang X, Zhang Y-KJ, Esterly N, Klaassen CD, Wan Y-JY. Gender disparity of hepatic lipid homoeostasis regulated by the circadian clock. J Biochem. 2009;145:609-623.

52. Monetti M, Levin MC, Watt MJ, et al. Dissociation of hepatic steatosis and insulin resistance in mice overexpressing DGAT in the liver. Cell Metab. 2007;6:69-78.

53. Neuschwander-Tetri BA. Hepatic lipotoxicity and the pathogenesis of nonalcoholic steatohepatitis: the central role of nontriglyceride fatty acid metabolites. Hepatology. 2010;52:774-788.

54. Postic C, Girard J. Contribution of de novo fatty acid synthesis to hepatic steatosis and insulin resistance: lessons from genetically engineered mice. $J$ Clin Invest. 2008;118:829-838.

55. Alkhouri N, Dixon LJ, Feldstein AE. Lipotoxicity in nonalcoholic fatty liver disease: not all lipids are created equal. Expert Rev Gastroenterol Hepatol. 2009;3:445-451.

56. Eichmann TO, Lass A. DAG tales: the multiple faces of diacylglycerol-stereochemistry, metabolism, and signaling. Cell Mol Life Sci. 2015;72:3931-3952.

57. Postic C, Dentin R, Girard J. Role of the liver in the control of carbohydrate and lipid homeostasis. Diabetes Metab. 2004;30:398-408.

58. Leach KL, Ruff VA, Wright TM, Pessin MS, Raben DM. Dissociation of protein kinase C activation and sn-1,2-diacylglycerol formation. Comparison of phosphatidylinositol- and phosphatidylcholine-derived diglycerides in alpha-thrombin-stimulated fibroblasts. J Biol Chem. 1991;266:3215-3221.

59. Lopez Cl, Pelletán LE, Suhaiman L, et al. Diacylglycerol stimulates acrosomal exocytosis by feeding into a PKC- and PLD1-dependent positive loop that continuously supplies phosphatidylinositol 4,5-bisphosphate. Biochim Biophys Acta. 1821;2012:1186-1199. 
60. Camporez JPG, Jornayvaz FR, Lee H-Y, et al. Cellular mechanism by which estradiol protects female ovariectomized mice from high-fat diet-induced hepatic and muscle insulin resistance. Endocrinology. 2013;154:1021-1028.

61. Anderson KE, Kielkowska A, Durrant TN, et al. Lysophosphatidylinositol-acyltransferase-1 (LPIAT1) is required to maintain physiological levels of Ptdlns and Ptdlns $P_{2}$ in the mouse. PLoS One. 2013;8:e58425.

62. D'Souza K, Epand RM. Enrichment of phosphatidylinositols with specific acyl chains. Biochim Biophys Acta. 1838;2014:1501-1508.

63. Yamashita A, Hayashi Y, Nemoto-Sasaki Y, et al. Acyltransferases and transacylases that determine the fatty acid composition of glycerolipids and the metabolism of bioactive lipid mediators in mammalian cells and model organisms. Prog Lipid Res. 2014;53:18-81.

64. Khan SA, Ali A, Khan SA, et al. Unraveling the complex relationship triad between lipids, obesity, and inflammation. Mediators Inflamm. 2014;2014:502749.

65. Extier A, Langelier B, Perruchot M-H, et al. Gender affects liver desaturase expression in a rat model of n-3 fatty acid repletion. J Nutr Biochem. 2010;21:180-187.

66. Kitson AP, Stroud CK, Stark KD. Elevated production of docosahexaenoic acid in females: potential molecular mechanisms. Lipids. 2010;45:209-224.

\section{SUPPORTING INFORMATION}

Additional Supporting Information may be found online in the supporting information tab for this article.

How to cite this article: Soares AF, Paz-Montoya J, Lei H, Moniatte M, Gruetter R. Sexual dimorphism in hepatic lipids is associated with the evolution of metabolic status in mice. NMR in Biomedicine. 2017;30:e3761. https://doi.org/10.1002/nbm.3761 